\title{
A NOTE ON THE RIESZ REPRESENTATION THEOREM
}

\author{
DON H. TUCKER
}

1. Introduction. In 1909, F. Riesz [5] gave an integral representation for the bounded linear transformations $T$ from the space of real valued continuous functions on $[0,1]$ into the real numbers, where the norm on the space is defined $\|f\|=\max |f(x)| ; 0 \leqq x \leqq 1$. An extensive bibliography for representation theorems is given in [1]. In 1961, S. E. James [2] generalized this result by considering continuous functions whose range of values was a subset of a Banach space $S$ and considered bounded linear transformations $T$ from this space into $S$. James' result required that the transformation $T$ be such that there exist a functional $\bar{T}$ from the real valued continuous functions on $[0,1]$ into the reals such that for each real valued continuous function $g$ on $[0,1]$ and for each $h$ in $S, T[g(x) h]=\bar{T}[g] \cdot h$.

The purpose of this note is to extend James' result in the following way: suppose $S_{1}$ is a linear normed space, $S_{2}$ is a Banach space, $C$ is the space of continuous functions from $[0,1]$ into $S_{1}$ with norm defined $\|g\|_{C}=\int_{0}^{1}\|g(x)\|_{S_{1}} d x$ and $B\left[S_{1}, S_{2}\right]$ is the space of continuous linear transformations from $S_{1}$ into $S_{2}$.

THEOREM 1. If $T$ is a bounded linear transformation from $C$ into $S_{2}$, then there exists a function $K$ defined and of bounded variation on $[0,1]$ with values in $B\left[S_{1}, S_{2}\right]$ such that, for each function $f$ in $C, T[f]$ $=\int_{0}^{1} d K(x) \cdot f(x)$.

2. Preliminary remarks. Continuity and bounded variation are considered as defined in the usual way with the appropriate norm used instead of absolute values. Since on the interval $[0,1]$ the HeineBorel theorem holds, each function in $C$ is bounded and uniformly continuous. Furthermore, if $f$ is in $C$, then $f_{n}(x)=\sum_{0=0}^{n}\left(\begin{array}{l}n \\ 0\end{array}\right) x^{v}(1-x)^{n-v}$ $\cdot f(v / n)$ converges uniformly and hence in norm to $f$. The argument in $[6$, p. 152] with absolute values replaced by norms goes through.

The integral used here is of the type defined by MacNerney [4]. The appropriate change of norm for absolute value in the argument in [6, p. 31] gives the following form of the Helly-Bray theorem: if $\left\{K_{n}(x)\right\}_{n=0}^{\infty}$ is uniformly of bounded variation on $[0,1]$ and $K_{n}(x)$ $\rightarrow K(x)$ as $n \rightarrow \infty$, the values of $K_{n}$ being in $B\left[S_{1}, S_{2}\right]$, then if $f$ is in $C$

Presented to the Society, January 22, 1962; received by the editors February 19 , 1962. 


$$
\lim _{n \rightarrow \infty} \int_{0}^{1} d K_{n}(x) \cdot f(x)=\int_{0}^{1} d K(x) \cdot f(x)
$$

\section{Proof of Theorem 1.}

LeMmA 1. If $G_{n}(x, t)=\sum_{v<n x}\left(\begin{array}{l}n \\ v\end{array}\right) t^{v}(1-t)^{n-v}$ for $0<x<1 ; 0 \leqq t \leqq 1$, and $k_{x}(t)=1$ for $0 \leqq t \leqq x ; k_{x}(t)=0$ for $x<t \leqq 1$, then $\int_{0}^{1}\left|k_{x}(t)-G_{n}(x, t)\right| d t$ $\rightarrow 0$ as $n \rightarrow \infty$.

Proof.

$$
\begin{aligned}
\sum_{|v| n-x \mid \geq \delta}\left(\begin{array}{l}
n \\
v
\end{array}\right) x^{v}(1-x)^{n-v} & \leqq \sum_{|v / n-x| \geq \delta}\left(\begin{array}{l}
n \\
v
\end{array}\right) x^{v}(1-x)^{n \rightarrow v} \frac{(n x-v)^{2}}{n^{2} \delta^{2}} \\
& \leqq \frac{1}{n^{2} \delta^{2}} \sum_{v=0}^{n}(n x-v)^{2}\left(\begin{array}{l}
n \\
v
\end{array}\right) x^{v}(1-x)^{n-v} \\
& =\frac{n x(1-x)}{n^{2} \delta^{2}}=\frac{x(1-x)}{n \delta^{2}} \leqq \frac{1}{4 \delta^{2} n}
\end{aligned}
$$

Now consider $G_{n}(x, t)=\sum_{v<n x}\left(\begin{array}{l}n \\ 0\end{array}\right) t^{v}(1-t)^{n-v}$ and take $t>(x+\epsilon)$. Then

$$
\begin{aligned}
\sum_{v / n<x}\left(\begin{array}{l}
n \\
v
\end{array}\right) t^{v}(1-t)^{n-v} & \leqq \sum_{t-v / n>\epsilon}\left(\begin{array}{l}
n \\
v
\end{array}\right) t^{v}(1-t)^{n-v} \\
& \leqq \sum_{|t-v / n|>e}\left(\begin{array}{l}
n \\
v
\end{array}\right) t^{v}(1-t)^{n-v} \leqq \frac{1}{4 \epsilon^{2} n}
\end{aligned}
$$

Now take $\epsilon=n^{-1 / 4}$; then $G_{n}(x, t)<n^{-1 / 2} / 4$ for $t>x+n^{-1 / 4}$, and so $G_{n}(x, t)$ converges uniformly to zero in every interval $x<t_{0} \leqq t \leqq 1$. From symmetry (i.e., consider $\left.1-G_{n}(x, t)\right) G_{n}(x, t)$ converges uniformly to 1 in every interval $1 \leqq t \leqq t_{0}<x$. The result then follows. The basic thought of this lemma is well known in the theory of probability. See comment by Lorentz [3, p. 4].

We shall denote by $C(R)$ the space of continuous real valued functions on $[0,1]$ with norm defined by $\|f\|_{C(R)}=\int_{0}^{1}|f(x)| d x$. Suppose $T$ is a bounded linear transformation from $C$ into $S_{2}$.

LEMMA 2. The transformation defined by $B_{g} \cdot k=T[g(x) \cdot k]$ for $g$ in $C(R)$ and $k$ in $S_{1}$ is, for fixed $g$, a bounded linear transformation from $S_{1}$ into $S_{2}$. Furthermore, $B_{g}$ is a bounded linear transformation from $C(R)$ into the Banach space $B\left[S_{1}, S_{2}\right]$. The latter statement holds whether we use the l.u.b. norm in $C(R)$ or the norm defined above. 
Proof. $B_{0}[\alpha k+\beta h]=T[g(x) \cdot(\alpha k+\beta h)]=\alpha T[g(x) \cdot k]+\beta T[g(x) \cdot h]$ and

$$
\begin{aligned}
\left\|B_{0} \cdot k\right\|_{S_{2}} & =\|T[g(x) \cdot k]\|_{S_{2}} \leqq|T| \cdot\|g(x) \cdot k\|_{c}=|T| \cdot \int_{0}^{1}\|g(x) \cdot k\|_{S_{1}} d x \\
& =|T| \int_{0}^{1}|g(x)| d x \cdot\|k\|_{S_{1}} \leqq[|T| \max |g|] \cdot\|k\|_{S_{1}} .
\end{aligned}
$$

Hence $\left\|B_{0}\right\| \leqq|T|\|g\|_{C(R)}$ whichever norm is used in $C(R)$. Furthermore,

$$
\begin{aligned}
\left(\alpha B_{0}+\beta B_{h}\right) \cdot k & =T[\alpha g(x) \cdot k]+T[\beta h(x) \cdot k]=T[\alpha g(x) \cdot k+\beta h(x) \cdot k] \\
& =T[(\alpha g(x)+\beta h(x)) \cdot k]=B_{\alpha g+\beta h} \cdot k .
\end{aligned}
$$

Hence $B_{0}$ is a bounded linear transformation from $C(R)$ into $B\left[S_{1}, S_{2}\right]$. We shall hereafter refer to this transformation from $C(R)$ into $B\left[S_{1}, S_{2}\right]$ as $]$.

Suppose $f$ is in $C$; then $f_{n}(x)=\sum_{v=0}^{n}\left(\begin{array}{l}n \\ v\end{array}\right) x^{v}(1-x)^{n-v} \cdot f(v / n)$ converges uniformly and in norm to $f$, and therefore $T\left[f_{n}\right]$ converges to $T[f]$. Also,

$$
T\left[f_{n}(x)\right]=\sum_{v=0}^{n} T\left[\lambda_{n, v}(x) \cdot f\left(\frac{v}{n}\right)\right]=\sum_{v=0}^{n} B_{\lambda_{n, v}} \cdot f\left(\frac{v}{n}\right)
$$

where

$$
\lambda_{n, v}(x)=\left(\begin{array}{l}
n \\
v
\end{array}\right) x^{v}(1-x)^{n-v} .
$$

Hence we may write $T\left[f_{n}\right]=\int_{0}^{1} d K_{n}(x) \cdot f(x)$, where $K_{n}(x)=\sum_{v<n x} B_{\lambda n, v}$, for $0<x<1 ; K_{n}(0)=N$, where $N$ denotes the transformation which maps $S_{1}$ into the zero point of $S_{2}$, and $K_{n}(1)=B_{1}$. Hence, for $0<x<1$, $K_{n}(x)=J\left[\sum_{v<n x} \lambda_{n, v}(t)\right] ; \sum_{v<n x} \lambda_{n, v}(t)=G_{n}(x, t)$ of Lemma 1 ; and for each $x$ this sequence converges in norm to $k_{x}(t)$ as $n \rightarrow \infty$. Since $J$ is a continuous transformation from $C(R)$ into $B\left[S_{1}, S_{2}\right]$ and $B\left[S_{1}, S_{2}\right]$ is complete, $K_{n}(x)$ converges for each $x$.

$$
\begin{aligned}
V_{0}^{1} K_{n}=\sum_{v=0}^{n}\left\|B_{\lambda_{n, v}}\right\| & \leqq \sum_{v=0}^{n}|T| \int_{0}^{1}\left|\lambda_{n, v}(x)\right| d x=|T| \sum_{v=0}^{n} \int_{0}^{1} \lambda_{n, v}(x) d x \\
& =|T| \int_{0}^{1} \sum_{v=0}^{n} \lambda_{n, v}(x) d x=|T|
\end{aligned}
$$

since $\lambda_{n, v}(x) \geqq 0$ for $0 \leqq x \leqq 1$ and $\sum_{v=0}^{n} \lambda_{n, v}(x) \equiv 1$. Therefore, $\left\{K_{n}\right\}$ 
are uniformly of bounded variation on $[0,1]$ and for each $x$ converge to some point $K(x)$ in $B\left[S_{1}, S_{2}\right]$, the function $K$ being of total variation not more than $|T|$ and then, by the Helly-Bray theorem, in $\$ 2$, $T[f]=\int_{0}^{1} d K(x) \cdot f(x)$.

4. Some remarks on the space $B\left[C, S_{2}\right]$. It is easily seen that for a given function $K$ of bounded variation on $[0,1]$ with values in $B\left[S_{1}, S_{2}\right]$ the transformation $T[f]=\int_{0}^{1} d K(x) \cdot f(x)$ is a linear transformation from $C$ into $S_{2}$ which is continuous if the uniform norm is used in $C$. It is also easy to see by way of examples that not all such transformations are continuous in the integral norm used above. (Let us assume that each $K$ considered has been minimized in total variation by defining $K(x)=\frac{1}{2}[K(x-)+K(x+)] ; 0<x<1$. This will not affect the transformation $T$ which it produces.) A natural question now would be, "For what functions $K$ is the corresponding $T$ continuous in the integral norm?" The answer is given by the following.

THEOREM 2. In order that $T[f]=\int_{0}^{1} d K(x) \cdot f(x)$ should be continuous in the integral norm it is necessary and sufficient that $K$ should satisfy a Lipschitz condition on $[0,1]$. Furthermore, the norm of the transformation $T$ is the g.l.b. of the Lipschitz constants for $K$.

Proof. The sufficiency being easily seen only the necessity will be proved here.

First, suppose $K$ is not continuous on $[0,1]$. Since $K$ is quasicontinuous and the total variation of $K$ has been minimized, there exists a point $p ; 0<p<1$ (if $p$ were 0 or 1 the argument need be only slightly changed) such that $K(p-) \neq K(p+)$ and a sequence of intervals $\left[p_{i}, q_{i}\right]$ such that $p_{i} \nearrow p$ and $q_{i} \searrow p$ and $K$ is continuous at the points $p_{i}$ and $q_{i}, i=0,1, \cdots$. Choose points $k_{i}$ in $S_{1}$ such that $\left\|k_{i}\right\|_{S_{1}}=1$ and

$$
\left\|\left[K\left(p_{i}\right)-K\left(q_{i}\right)\right] k_{i}\right\|_{s_{2}} \geqq\left\|K\left(p_{i}\right)-K\left(q_{i}\right)\right\|-\frac{1}{i}
$$

and define

$$
\begin{aligned}
g_{i}(x) & =N_{S_{1}}\left(\text { the zero point of } S_{1}\right) 0 \leqq x \leqq p_{i}, q_{i} \leqq x \leqq 1 \\
& =k_{i} \quad \text { otherwise. }
\end{aligned}
$$

Then $\left\|g_{i}(x)\right\|=\left\|k_{i}\right\| \cdot\left(q_{i}-p_{i}\right) \rightarrow 0$ as $i \rightarrow \infty$ and furthermore $\int_{0}^{1} d K(x)$ $\cdot g_{i}(x)$ exists. Now choose $f_{i} \in C$ so that $\left\|f_{i}-g_{i}\right\|_{C}<1 / i$ and hence 
$\left\|f_{i}\right\|_{c} \rightarrow 0$. Furthermore $\left\|\int_{0}^{1} d K \cdot\left[f_{i}-g_{i}\right]\right\|_{S_{2}} \rightarrow 0$ but $\int_{0}^{1} d K \cdot g_{i}$ $=\left[K\left(q_{i}\right)-K\left(p_{i}\right)\right] \cdot k_{i}$ so that

$\left\|\int_{0}^{1} d K \cdot g_{i}\right\|_{S_{2}} \geqq\left\{\left\|K\left(p_{i}\right)-K\left(q_{i}\right)\right\|-\frac{1}{i}\right\} \rightarrow\|K(p+)-K(p-)\|>0$, so that $\left\|\int_{0}^{1} d K \cdot f_{i}\right\|_{s_{2}} \rightarrow\|K(p+)-K(p-)\|>0$, but $\left\|f_{i}\right\|_{c \rightarrow 0}$. Hence $K$ is continuous.

Second, suppose $K$ is not Lipschitz on $[0,1]$. Then there exists a sequence $\left[p_{i}, q_{i}\right]$ of subintervals of $[0,1]$, whose lengths converge to zero and such that $\left\|K\left(q_{i}\right)-K\left(p_{i}\right)\right\|>i\left(q_{i}-p_{i}\right)$. Define $g_{i}(x)$ $=1 /\left(q_{i}-p_{i}\right) \cdot 1 / i \cdot k_{i}$ for $p_{i} \leqq x \leqq q_{i}$ where $k_{i}$ is a point in $S_{1}$ of norm 1 for which $\left\|\left[K\left(q_{i}\right)-K\left(p_{i}\right)\right] \cdot k_{i}\right\|_{S_{2}}>i\left(q_{i}-p_{i}\right)$ and $g_{i}(x)=N_{S_{1}}$ elsewhere. $\left\|\int_{0}^{1} d K \cdot g_{i}\right\|_{S_{2}}>1$ and $\left\|g_{i}\right\|=1 / i$. Approximate $g_{i}$ with $f_{i}$ in $C$ as before and obtain a contradiction which establishes the first statement of the theorem. The final statement of the theorem then follows readily by a similar argument.

\section{BIBLIOGRAPHY}

1. N. Dunford and J. T. Schwartz, Linear operators. I, Interscience, New York, 1958.

2. S. E. James, Integration in normed linear spaces, M.S. Thesis, University of Utah, Salt Lake City, Utah, 1961.

3. G. G. Lorentz, Bernstein polynomials, Univ. of Toronto Press, Toronto, 1953.

4. J. S. MacNerney, Stieltjes integrals in linear spaces, Ann. of Math. (2) 61 (1955), 354-367.

5. F. Riesz, Sur les operations fonctionnelles linéaires, C. R. Acad. Sci. 1949 (1909), 974-977.

6. D. V. Widder, The Laplace transform, Princeton Univ. Press, Princeton, N. J., 1946.

UNIVERSITY OF UTAH 